\title{
Stent entrapment and guide wire fracture during percutaneous coronary intervention in the same patient
}

\author{
Batuhan Tamci', Taha Okan², Hasan Gungor ${ }^{3}$, Volkan Ozturk', Fatih Cam ${ }^{4}$ \\ 1Department of Cardiology, Sifa University, Izmir, Turkey \\ 2Department of Cardiology, Kardiya Medical Center, Izmir, Turkey \\ 3Department of Cardiology, Adnan Menderes University, Aydin, Turkey \\ ${ }^{4}$ Department of Cardiology, Medline Hospital, Aydin, Turkey
}

Postep Kardiol Inter 2013; 9, 2 (32): 190-193

DOI: $10.5114 /$ pwki.2013.35459

\begin{abstract}
In the performance of increasingly complex PCI there remains an ever-present risk of stent entrapment and guide wire or other device fracture. We report the first case with stent dislodgement and guide wire fracture to occur simultaneously in the same patient.
\end{abstract}

Key words: guide wire fracture, stent entrapment.

\section{Introduction}

In the performance of increasingly complex percutaneous coronary intervention $(\mathrm{PCl})$ there remains a risk of stent entrapment and guide wire or other device fracture [1]. Entrapment of the stent or guide wire in coronary arteries is a rare but life-threatening complication of $\mathrm{PCl}$, which can result in intracoronary or systemic embolization, thrombus formation, emergent coronary artery bypass graft surgery, or death. The incidence of stent or other device loss during $\mathrm{PCl}$ has significantly declined in recent years, from $8.3 \%$ to $0.21 \%$, probably due to improvements in equipment design and technology with the universal use of premounted stents [1-3]. Different retrieval techniques of unexpanded stents have been implemented with a success rate as high as $86 \%$. These techniques include use of balloon catheters (inflation within or distal to the lost stent), myocardial biopsy and biliary forceps, two twisted guide wires, basket devices, loop snares, etc. Nevertheless, surgical removal can still be required in a few cases [1-4]. Also guide wire fractures during $\mathrm{PCl}$ are extremely rare and occur in approximately $0.02-0.08 \%$ of cases [3, 4]. Guide wire remnants can lead to an acute ischemic event due to thromboembolic occlusion and perforation $[5,6]$. Surgical management, percutaneous removal techniques, stent implantation over the guide wire remnants, or conservative follow-up can be chosen [3, 6-8].
We report a case of a patient with a stripped stent during $\mathrm{PCl}$, which was conducted by another invasive center, and the patient was subsequently transferred to our center to treat this complication. The stripped stent had been deployed successfully using modified small balloon technique. However, guide wire fracture occurred during $\mathrm{PCl}$. The second complication was treated by moving the guide wire remnant to the distal part of the coronary artery and embedding it by stent implantation over the wire. To our knowledge, this is the first case reported on stent dislodgement and guide wire fracture to occur simultaneously in the same patient.

\section{Case report}

A 73-year-old female patient was admitted to another hospital with acute coronary syndrome. Selective coronary angiography revealed an $80-85 \%$ stenosis in the left circumflex coronary artery (LCX). The hospital's interventional cardiologists planned to treat the patient with a bare metal stent (BMS) after predilatation. They attempted to insert the stent into the LCX but failed to cross the atherosclerotic plaque due to significant proximal angulation and severe vessel calcification. During these manipulations the balloon was stripped from the stent and the patient was immediately transferred to our center to treat this complication. There was typical angina pectoris, and ST segment

\section{Corresponding author:}

Hasan Gungor MD, Department of Cardiology, Faculty of Medicine, Adnan Menderes University, 09100 , Aydin, Turkey, tel.: +90 5068892238 ,

e-mail: drgungorhasan@yahoo.com

Received: 4.01.2013, accepted: 9.05.2013. 
depression when she was admitted to our catheter laboratory. Selective coronary angiography revealed the unexpanded stent embedded in the proximal part of the LCX (Figure 1). We inserted a new guide wire, crossed the unexpanded stent and placed this guide wire in the distal LCX. Over this guide wire we inserted a $1.5 \mathrm{~mm} \times 10 \mathrm{~mm}$ low profile angioplasty balloon catheter through the inside of the stripped stent. The stent was attached to the retrieval balloon by inflation to 5-6 atm and was then retracted into the guiding catheter but the embedded stent could not be removed and the patient had angina pectoris. We decided to deploy an unexpanded stent in the LCX by a balloon catheter urgently, managed by compressing the inflated slipped stent against the vessel wall with another $3.0 \mathrm{~mm}$ $\times 15 \mathrm{~mm}$ BMS, without compromising coronary blood flow (Figure 2). At the end of $\mathrm{PCl}$, during crossing, the hydrophilic coated non-metallic tip of the guide wire (PT2, Boston Scientific Corporation) became detached and was fractured by stent striates (Figure 3). We decided to retrieve the guide wire remnant by twisted guide wire technique but this technique failed. The guide wire was pushed to the distal segment of the LCX by a small, under-inflated balloon catheter and the retained guide wire tip was isolated from the circulation by implantation of a $2.5 \mathrm{~mm} \times 12 \mathrm{~mm}$ BMS over the wire (Figure 4). After 1 week optical coherence tomography (OCT) was performed to control the stent struts and OCT revealed multiple thrombi in the struts and thrombosed area near the distal part of the stent (Figure 5 ). We inserted a $3.5 \mathrm{~mm} \times 24 \mathrm{~mm}$ drug-eluting stent (DES) in the old stent covering the thrombosed area. After 3 days, the pa-

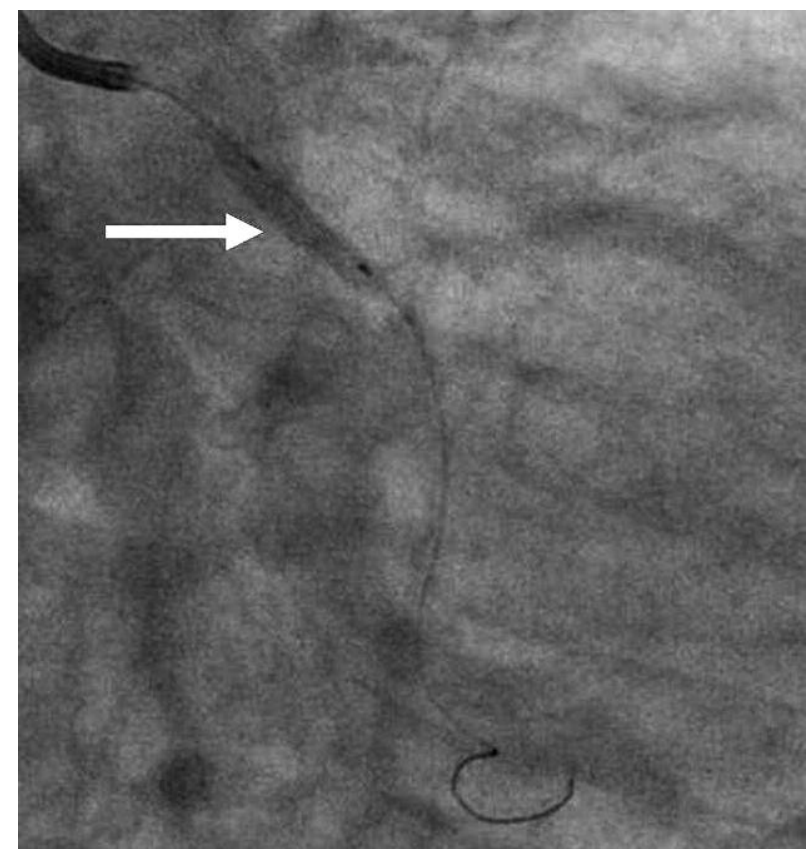

Fig. 2. Unexpanded stent in the LCX managed by compressing the inflated slipped stent against the vessel wall with another $3.0 \mathrm{~mm} \times 15 \mathrm{~mm}$ bare metal stent

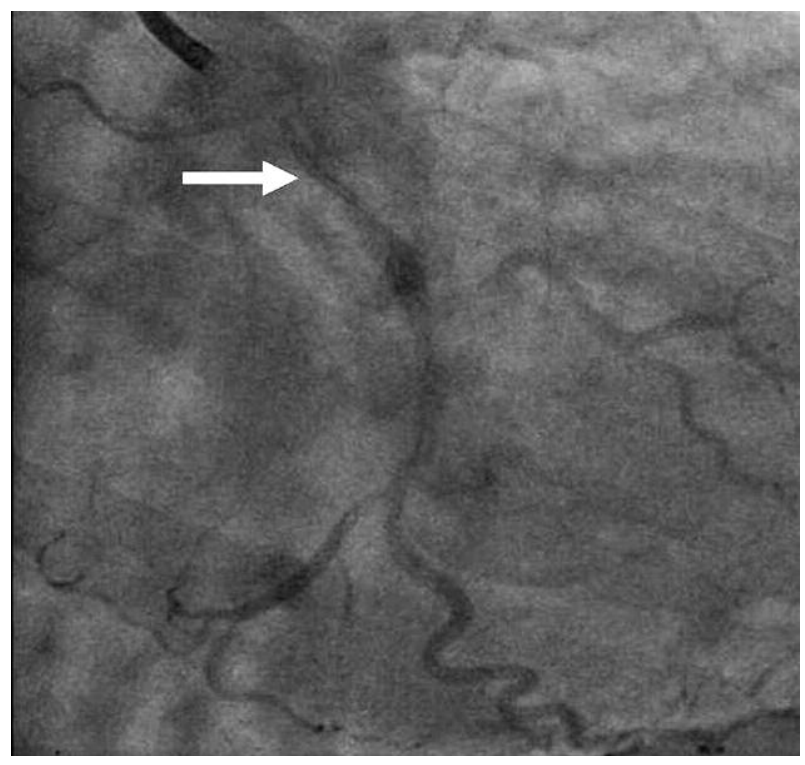

Fig. 1. Selective coronary angiography demonstrating unexpanded stent embedded in the proximal part of the circumflex artery

tient was discharged on oral medication including an ACE inhibitor, aspirin, $\beta$-blocker, clopidogrel and statin. She did not experience any thromboembolic events or symptoms over 1-month clinical follow-up.

\section{Discussion}

The incidence of stent or other device loss during PCI has significantly declined in recent years, probably due to

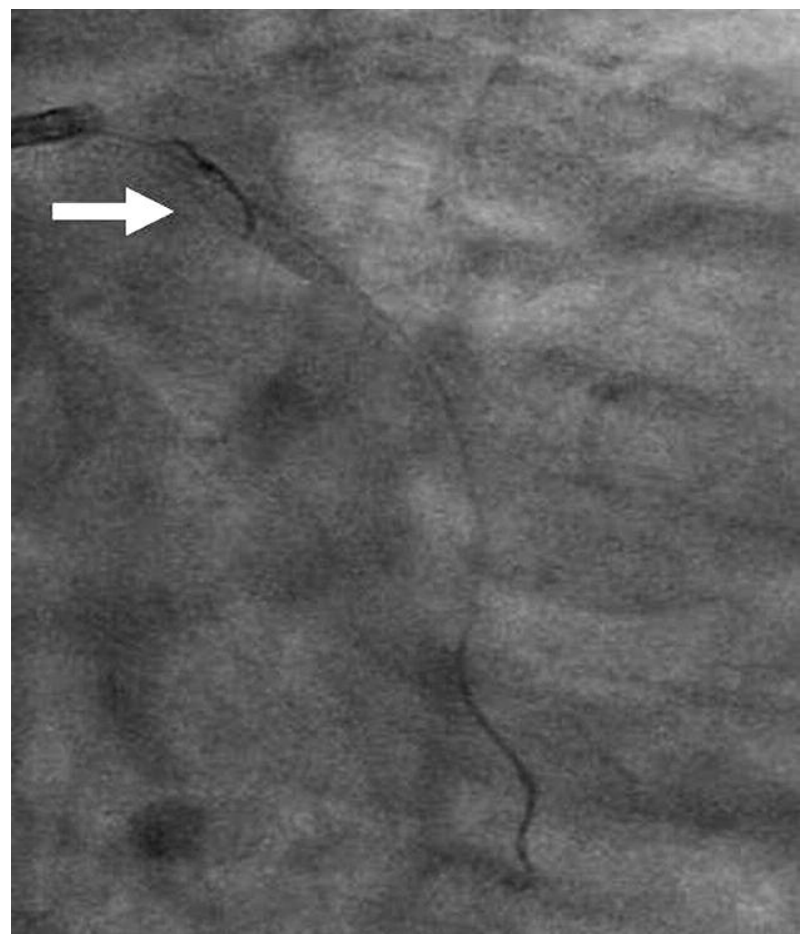

Fig. 3. Detached and fractured hydrophilic coated non-metallic tip of the guide wire by stent struts 


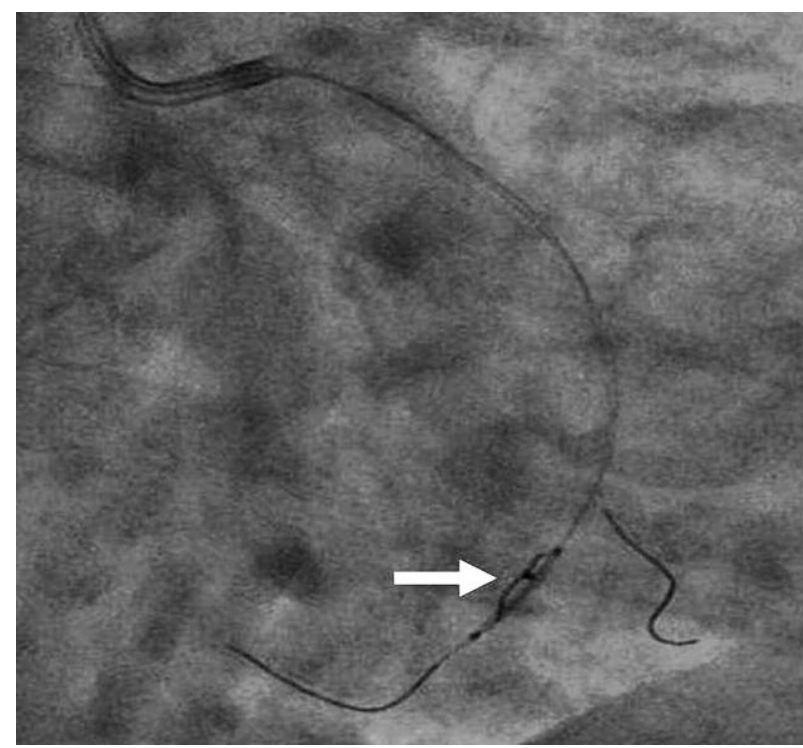

Fig. 4. The guidewire was pushed to the distal segment of the circumflex artery by a small, underinflated balloon catheter and it was isolated from the circulation by a $2.5 \mathrm{~mm} \times 12 \mathrm{~mm}$ bare metal stent implanted over the wire

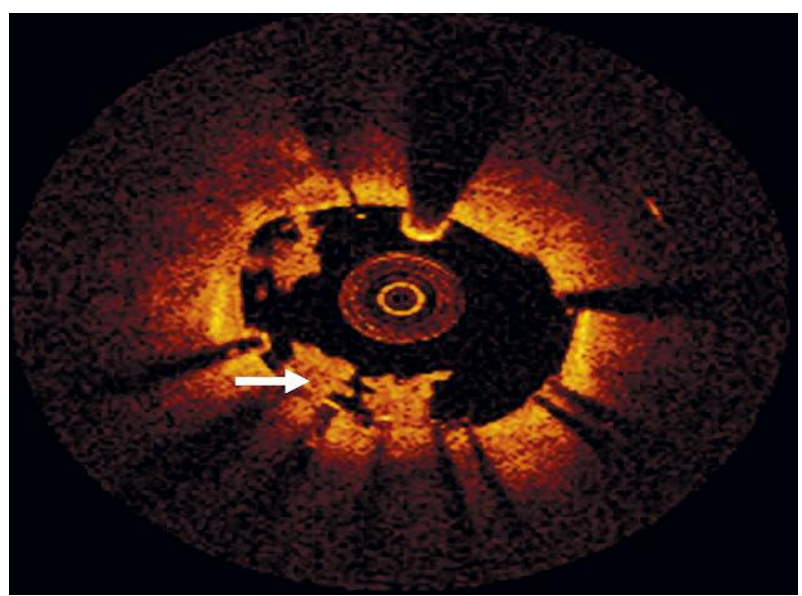

Fig. 5. Optical coherence tomography demonstrating multiple thrombi in the struts and thrombosed area near the distal part of the stent

improvements in equipment design, technology and universal use of pre-mounted stents [2, 3]. Stents were the most commonly lost devices and nowadays stent loss incidence is as low as $0.21 \%$ [3]. The most common cause of stent loss in series was attempts to deliver a stent through a previously deployed stent $[3,8]$ or when the stent-balloon assembly was pulled back into the guiding catheter before the target lesion [1, 2]. Poor support of the guiding catheter or guide wire, vessel tortuosity proximal to the lesion or severe vessel calcification and long stent may predispose to stent entrapment [1-3]. In our case, the LCX was heavily calcified and had significant angulation.

A stripped stent may cause intracoronary or systemic embolization, thrombus formation, emergent coronary artery bypass graft surgery, or death [1, 2]. Several techniques for retrieval of unexpanded stents from the coronary artery have been described with a success rate as high as $86 \%$, including use of balloon catheters (inflation within or distal to the lost stent), myocardial biopsy and biliary forceps, two twisted guide wires, basket devices, and loop snares; nevertheless, surgical removal is required in a few cases [1-4]. The most commonly used technique was the small balloon technique, which is the simplest retrieval technique [2]. This technique requires a retained guide wire in the slipped stent which allows advancement of the retrieval balloon into the stent entrapped on it [1, 2]. Our patient was admitted to our catheter laboratory without a guide wire. We inserted a new guide wire and crossed the unexpanded stent, but the guide wire could not be crossed throughout the stent's true lumen and the retrieval balloon could not attach the entrapped stent adequately. For this reason we could not remove the embedded stent.

If the stent is lost from the wire, intracoronary stent entrapment is managed by compressing the unexpanded stents against the vessel wall with another stent or by crushing [1, 2]. Crushing or deploying the stent should probably be avoided if the stent is located in the left main coronary artery, proximal left anterior descending artery or other critical location. Crushing or deploying the stent carries a risk of restenosis. Meticulous attention should be given to completely apposing the stent struts with the vessel wall to avoid limitation of blood flow through the coronary artery, which can be confirmed by intravascular ultrasound $[1,9]$. In our case OCT of the LCX demonstrated a wellexpanded stent; the struts of the undeployed stent were well visualized, and were compressed against the vessel wall without dissection. But multiple thrombosed areas in the struts were noted. The use of OCT in this case assured the authors that the stent was well deployed and adequately expanded to prevent complications such as stent thrombosis and restenosis. Furthermore, the use of OCT enabled the authors to rule out dissection of the vessel wall and to evaluate the status of the crushed stent. The present case demonstrates that the technique of crushing an undeployed stent combined with OCT use is remarkably easier and faster than a number of tedious stent retrieval techniques. It could be used safely and effectively in preference to a number of challenging stent retrieval techniques.

Complete fracture of a guide wire during $\mathrm{PCl}$ is a very rare event. It can lead to severe clinical outcomes due to thrombosis and occlusion of the coronary vessel, perforation and systemic embolism risk. The management can be interventional, surgical or conservative, depending on the clinical situation of the patient and the position of the guide wire remnants in the coronary artery $[3,5,6]$.

A few authors preferred leaving the guide wire remnant within the coronary vessel, especially in patients who have a high risk for surgery. However, the coronary seg- 
ment that contained the guide wire remnant showed progressive stenosis in subsequent angiography. Small fragments of wire may be left within the coronary artery without adverse sequelae, especially if they are contained within small, chronically occluded coronary vessels or within a distal part of the vessel [10].

Various methods for retrieval of guide wire fragments are available. Snares of various types have been utilized including gooseneck snares and loop snares, which are more suitable for proximal, large-caliber vessels [6, 7]. Two or three wire rotation over the retained wire for removal of a fractured angioplasty guide wire is suitable for retrieval in small and medium sized vessels [7]. Another technique is isolating the guide wire remnants from the circulation with a stent procedure [6]. In our case, the remnant guide wire tip was moved down to the distal segment of the LCX by a small, under-inflated balloon catheter and the retained guide wire tip was fixed by stent implantation.

\section{Conclusions}

Guide wire fracture and stent dislodgement are uncommon. Stent implantation over the guide wire remnants and entrapped stents can be chosen. This is the first reported case of stent dislodgement and guide wire fracture to occur simultaneously in the same patient.

\section{References}

1. Brilakis ES, Best PJ, Elesber AA, et al. Incidence, retrieval methods, and outcomes of stent loss during percutaneous coronary intervention: a large single-center experience. Cathet Cardiovasc Intervent 2005; 65: 333-340.

2. Eggebrecht $\mathrm{H}$, Haude $M$, von Birgelen $\mathrm{C}$, et al. Nonsurgical retrieval of embolized coronary stents. Cathet Cardiovasc Intervent 2000; 51: 432-440.

3. Iturbe JM, Abdel-Karim AR, Papayannis A, et al. Frequency, treatment, and consequences of device loss and entrapment in contemporary percutaneous coronary interventions. J Invasive Cardiol 2012; 24: 215-221.

4. Khonsari S, Livermore J, Mahrer P, et al. Fracture and dislodgment of floppy guidewire during percutaneous transluminal coronary angioplasty. Am J Cardiol 1986; 58: 855-856.

5. Patel T, Shah S, Pandya R, et al. Broken guidewire fragment: a simplified retrieval technique. Cathet Cardiovasc Intervent 2000; 51: 483-486.

6. Karabulut A, Dağlar E, Çakmak M. Entrapment of hydrophilic coated coronary guidewire tips: which form of management is best? Cardiol J 2010; 17: 104-108.

7. Collins N, Horlick E, Dzavik V. Triple wire technique for removal of fractured angioplasty guidewire. J Invasive Cardiol 2007; 19: E230-E234.

8. Balbi M, Bezante GP, Brunelli C, et al. Guide wire fracture during percutaneous transluminal coronary angioplasty: possible causes and management. Interact Cardiovasc Thorac Surg 2010; 10: 992-994.

9. Wongpraparut N, Yalamachili V, Leesar MA. Novel implication of combined stent crushing and intravascular ultrasound for dislodged stents. J Invasive Cardiol 2004; 16: 445-446.
10. Alexiou K, Kappert U, Knaut M, et al. Entrapped coronary catheter remnants and stents: must they be surgically removed? Tex Heart Inst J 2006; 33: 139-142. 